\title{
GEO-LOCATING HISTORICAL SURVEY DATA AND IMAGES - A CASE STUDY FOR THE CANNING RIVER, PERTH, WESTERN AUSTRALIA
}

\author{
P. Helmholz ${ }^{1}$, Y. Mousa ${ }^{1,2}$, T. Snow ${ }^{1}$, A. Haebich ${ }^{1}$, G. Piggot ${ }^{1,3}$, J. Tonkin $^{1}$, W. Lamont ${ }^{1}$ \\ ${ }^{1}$ Curtin University, GPO Box U1987, Perth WA 6845 (petra.helmholz, mousa.yousif, t.snow, a.haebich, \\ wesley.lamont)@curtin.edu.au; (guy.piggott, jack.tonkin)@graduate.curtin.edu.au \\ ${ }^{2}$ Civil Engineering Department, Al-Muthanna University, Al-Muthanna, Iraq \\ ${ }^{3}$ BHP, Level 37, 125 St Georges Terrace, Perth, WA, 6000, Australia, guy.piggott@bhp.com
}

\section{Commission VI, WG IV/9}

KEY WORDS: Historical maps, Western Australia, Archives, Unity, geo-visualisation

\begin{abstract}
:
The aim of this project is to create the required framework to allow the transformation of the Canning River location survey data captured by Dr. J. A. Ludwig Preiss from 1841 into today's maps and to utilise visualisation techniques to analyse the results. The original survey data includes distances and bearing observations as well as 14 historical maps. Firstly, the old survey data (includes distances and angles measurements) is plotted into a local coordinates system using modern surveying software (MAGNET Office). Then, common points (unchanged locations) are identified by comparison with the plotted and the current paths of the river. A similarity and affine transformation are used to find transformation parameters that allow to geo-locate the plotted river into the current geodetic datum (MGA94). The calculated Root Mean Squared Errors (RMSE) are $21.7 \mathrm{~m}$ and $21.1 \mathrm{~m}$ obtained by geo-locating the common points using similarity and affine transformation, respectively. For geo-referencing the historical maps, the similarity, projective and Thin Plate Spline (TPS) transformations have been applied. It has been found that one point of interest (referred to as Nairn's house), which was drawn in one of the historical maps, still exists today (now known as Maddington Homestead). The distances from the actual position of Nairn's house to its position in the georeferenced maps using similarity, projective and TPS are $11.8 \mathrm{~m}$, $13 \mathrm{~m}$, and $14 \mathrm{~m}$ respectively. All the gained information and map details are utilised in creating a dynamic visualisation suitable for comparing the generated map and historical map with modern aerial imaging and DTM data.
\end{abstract}

\section{INTRODUCTION}

Historical maps have been used and shown to be of importance, not just for historic purposes, but also for several different fields including urban planning, field cultivation and landcover/ land use (LCLU) changes (Király et al., 2008). Historical maps help to explain the evolution of areas and their dynamics over time. If the aim is to extract complex information, the precise georeferencing of the maps is important. This paper focuses on original field observations and maps produced during a survey along the Canning River in Western Australia. They have been identified to be of great value because of the historical information of landmarks such as homesteads, significant trees, and information about the flora noted in the field sheets and on the maps.

The 1841 survey of the Canning River was commissioned by Governor Hutt of the Colony of Western Australia to record existing land grants and sites for new land settlement. Hutt appointed the German naturalist, Dr. Johann August Ludwig Preiss (1811-1883, who arrived in the colony in 1838 to lead the survey (Calaby, J. H, 1967). It was common practice to use naturalists and other men of science due to the lack of trained surveyors in the Australian colonies.

The survey was a Crown Grant Survey designed to define the actual boundaries of each grant. As per the practices of the day the original crown grant boundary posts may not have been placed by a surveyor, but the grant holder himself. Preiss may have only surveyed the line between the marks. The original Crown Grant Marks are still accepted today as being the defining point regardless of later dimensions.
The survey was completed observing the left bank of the river only, as well as the location of property boundaries. Overall, there are 2 field books available; the first field book is related to the mapping of the Canning River, while the second field book is related to the mapping of properties. Each field book contains more than 90 pages/ field sheets. The field sheets contain more information than just the survey data. This additional information is important material for historians and includes the location of homesteads, points of interests (creeks entering the river and similar) as well as botanical information recorded in field sheets.

During the 1840 s a series of maps were produced based on the field sheets. Overall, there are 14 maps focusing on the location of the Canning River. The available survey data in the first field book covers nearly $2 \mathrm{~km}$ of the river and relates to three maps. The other 11 maps available also show part of the river and other objects of interest such as property boundaries.

The State Records Office (SRO) of Western Australia has scanned the historical field sheets as well as maps in order to preserve them. These digital files were available to us as part of the project. This paper focuses only on the field sheets and maps containing information about the Canning River.

The objectives of the project are:

To redraw the historical map based on the original field sheets

To reference the redrawn map with the historical maps and to compare those data

To geo-reference the re-drawn river map as well as the historical maps using Ground Control Points (GCPs) picked along the river and to assess the accuracy of the georeferencing process

\footnotetext{
* Corresponding author
} 
- To identify a small number of points of interest and to utilise in the accuracy assessment

- $\quad$ To present the information in a format in which non-spatial experts can see the process as well as the final results.

The paper is structured as follows: Section 2 provides more details about related work and Preiss' survey including the methods used, and the results of the survey. The data sources which have been used for the geo-referencing will be further introduced. Section 3 reviews the methods which have been used during the geo-referencing process and the visualisation processes and techniques and their utilisation. Section 4 presents the project results, including a detailed analysis of the results utilising the different transformation methods. Section 5 concludes the paper.

\section{BACKGROUND}

\subsection{Related Work}

Extensive research has been undertaken in the field of georeferencing historical ortho-images or historical images. Often, the bundle adjustment using unchanged topographic features as Ground Control Points (GCP) is used in a multi-scale approach. New points are obtained from the block adjustment of the lower resolution images that can be used as control points in the block adjustment of the next scale (Zhu at el., 2008). This is an iterative process and is repeated until all resolution levels have been processed. In some cases, line features such as road network and building limits are used instead of point features (Cléry et al., 2014). In order to enhance the accuracy, sometimes additional information is used, including Digital Surface Models (DSM) derived from the historical images (Giordano, et al., 2018) or distance, orientation difference and overlap between matched lines are used. The ortho-images and images used often date no further back then the 1930s (Cléry et al., 2014), the 1940s (Giordano, et al., 2018) or even the 1960s/1980s (Zhu, et al., 2008). Giordano, et al. (2018) contains a good overview of photogrammetric and non-photogrammetric methods used in this context.

A much greater challenge is presented when attempting to georeference historical maps. A problem which historical orthoimages/map and topographic maps have in common, is the process of the scanning. Care must be taken when scanning the maps, i.e. using a calibrated large-format scanner, appropriate resolution, and image file format (Affek, 2013).

Nevertheless, historical satellite and airborne images are "young" compared to some historical topographic maps. Due to the age of some of these maps, the used projection and geodetic referencing systems are often unknown (Király et al., 2008).

If the used geodetic referencing system and the map projection are known, the transformation of the historical map into today's datum can be performed using the known parameters (Király et al., 2008). The challenges for this kind of transformation are well explained in (Affek, A., 2013). Furthermore, if the historical map is based on geodetic measurements (e.g. triangulation networks) and a geocentric geographic coordinate system with graticules or measured grids is used, then usually a similarity of affine transformation is used (Affek, A., 2013).

If the geodetic referencing system and the map projection are unknown, and the maps were not created based on detailed geodetic measurements but rather on estimated distances and angles, transformation parameter will have to be determined using GCPs. The selection of GCPs is usually easy and possible in topographic maps rich in information and features which do not change over time. For instance, mountain ranges, historical roads and property boundaries. However, not all historical maps are information rich.

Topographic maps produced up to the end of the 18th century often have an unknown geodetic referencing system and map projection and have not been observed using geodetic networks. In this case a polynomial transformation and/or a rubber-sheet method are appropriate. As explained in Király et al. (2008), the rubber-sheet transformations are a group of transformations, where residual errors do not exist; the control points are transformed exactly to the to-coordinates of the points. There are some discrepancies how the transformation vectors are interpolated between the control points. The most common way is based upon the Delaunay triangulation.

Király et al. (2008) applied both transformation methods (a polynomial transformation and a rubber-sheet method) to georeference historical topographic maps dating back to the period from 1780-1826. The maps have been produced by the Military Survey of the Austria and have a relatively large scale of $1: 12,000$. The mean point error was the largest $(41.9 \mathrm{~m})$ when using the $3^{\text {rd }}$ order polynomial transformation and improved using the non-linear rubber sheeting method (38.4) and the linear rubber sheeting method $(24 \mathrm{~m})$.

If unprocessed (raw) data is available (e.g. field sheets and notes), the challenge of different map projection and cartographic generalisation does not exist. Cartographic generalisation is the effect based on the cartographer's interpretation and adjusted to a map's scale and application (cartographic and thematic generalisation) necessary to visualise all required information on a map. Cartographic generalisation could be a source to introduce errors in the geo-referencing process. The cartographic generalisation is generally low in maps which were created out of pure cartographic interest (e.g. military maps) compared to maps which has been produced for public purposes (Balletti, 2006).

It is also important to make the geo-referenced historical maps available to the public (Previtali, 2017) and to present them in museums exhibitions in an interesting way. There are several agencies making geo-reference historical data available, e.g. projects carried out by several National Geographical Institutes. For instance, in France the IGN published the "Carte d'ÉtatMajor"(www.geoportail.gouv.fr/donnees/carte-de-letat-major1820-1866). Another project is the Divenire project by the National archive Milan (www.asmilano.it/Divenire/home.htm). After the data is geo-referenced and publicly available, the extraction of explicit map information, e.g. the location and areas of settlement is the next challenge, already addressed in several papers (Herold et al., 2011).

The resulting data, points of interest and historical information is process into a dynamic three-dimensional visualisation. Overlaying the generated map, historical map and historical points of interest with modern aerial photography and digital terrain models (DTM) will allow for the contrasting of the historic with modern imagery and reveal not only the modern equivalent of historic points of interest but also the topographical influence on the Canning River's historical variance.

\subsection{Surveying methods used by Preiss}

The Canning River survey was observed using a series of traverse lines with chain and bearing measurements. For distance observations links and chains were used. A chain consisted of 100 links and converts to $20.117 \mathrm{~m}$ in today's metric system. The chain would be pulled until tense ensuring there were no kinks, 
from the starting position to the end to record a distance measurement. Chain measurements were to be done at a horizontal level otherwise a correction for the slope had to be applied. Generally flat open ground was required to avoid the chain catching on obstructions.

Along with the chain, a circumferentor was used to perform direction observations. A circumferentor is a survey compass which would have been placed on top of a tripod. By lining up the target point through the eye pieces, angle readings were recorded. All angle readings in the Canning River survey were observed to the nearest minute, which was the accuracy the survey compass could be observed to.

As the Canning River survey was completed over $20 \mathrm{~km}$ of riverbank, the task surveyor Ludwig Preiss had of keeping the survey as accurate as possible in rugged conditions is immense.

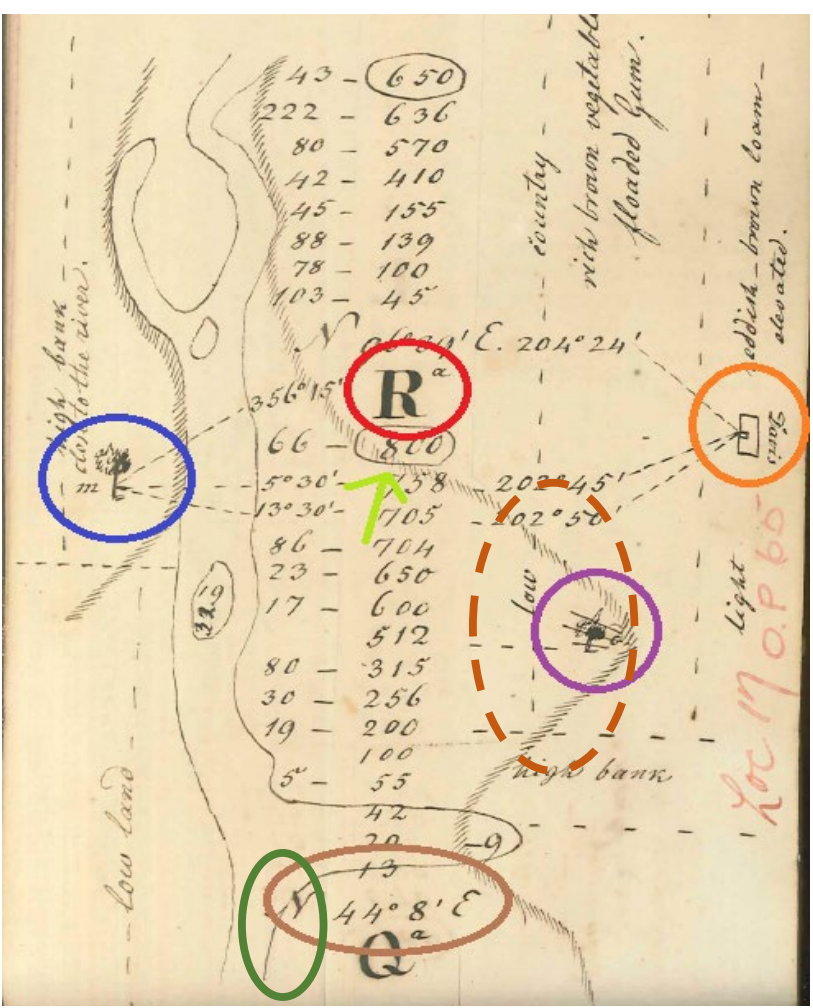

Figure 1: Field sheet of Preiss' survey of the Canning River.

It was possible to re-draw the location of the river and any points of interest using a standard surveying software (in this case MAGNET (Topcon, 2020)).

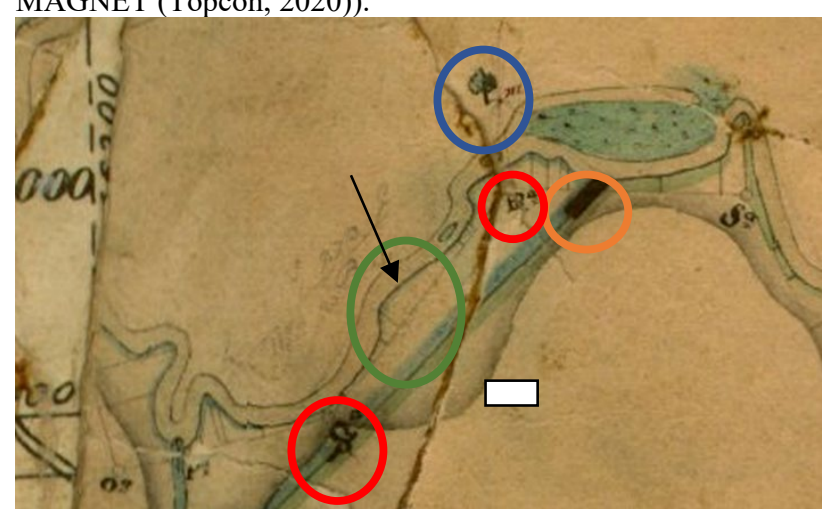

Figure 2: Section of the historical map fitting the field sheet provided in Figure 1.
A field sheet example is presented in Figure 1. The red circle shows an example of a station. Here the station is called Ra. The solid brown circle contains the azimuth from station Qa to station $\mathrm{Ra}$. In this case the azimuth is $44^{\circ} 8^{\prime}$. Please note, that the azimuth (relative to magnetic north) and not the bearing (relative to grid/map north) are observed. The magnetic declination in this area is $5^{\circ}$ and had been applied manually when producing the historical maps. Furthermore, the distance observations (chains) are indicated with the light green arrow in Figure 1. The offsets to the river, also in chains, are indicated with a dark green circle. If distance measurements were not possible, additional azimuth were observed enabling the determination of a location using the resection method. Such side angle readings are highlighted with the dashed brown circle. Furthermore, the orange circle is an example of a cottage located along the survey, the blue circle being a tree that was also located. The purple circle indicates a low point of the river.

The section of the river in one of the historical maps which belongs to the field sheet showing in Figure 1 is presented in Figure 2. Station Qa and $\mathrm{Ra}$ are both highlighted with a red circle. Furthermore, the tree which was highlighted in blue in Figure 1 is also highlighted in blue in Figure 2. The left-hand side of the river is indicated by a black arrow, and the offsets which were used to draw the river are highlighted with a green circle. While the maps are generally well preserved, there are some sections which are of poor quality due to the age of the maps. In addition, not all information has been transferred to the maps. For instance, the building highlighted in orange in Figure 1 is missing. The approximate location of this building is shown in Figure 2 (white box).

While the magnetic declination is indicated in the maps with $5^{\circ}$ no further information about any projection is used. Therefore, also considering the overall size of the survey area, it is assumed that a planar project has been used.

\subsection{Information used during geo-referencing}

Two methods of geo-referencing have been identified. Firstly, some of the historical maps also contain information about property boundaries (see highlighted note in Figure 3). Those property boundaries are also shown in the map including markers for the end of a chain (red arrow in Figure 4). Those markers were also established in the field. Furthermore, the maps contain the information of the property owners as well as the approximate size of the property (blue arrow in Figure 4)

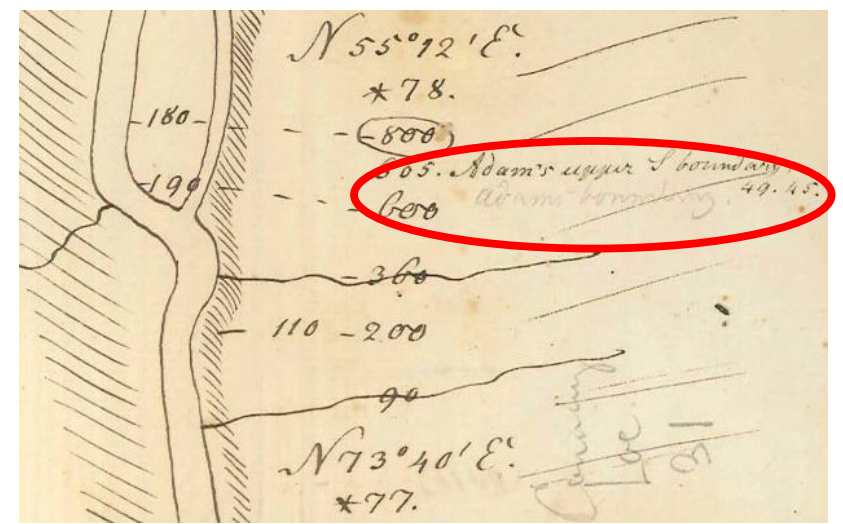

Figure 3: Field notes showing a property boundary (highlighted in red). 


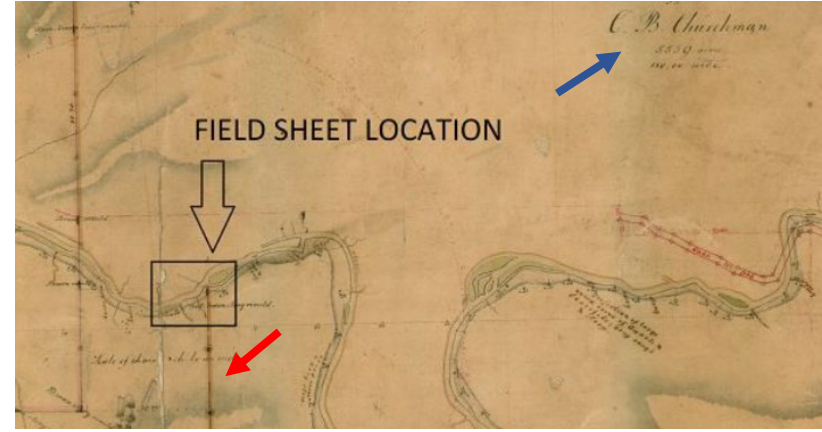

Figure 4: Historical map including property boundaries.

The most common way of changing property boundaries in Perth is through subdivisions. Therefore, the property boundaries (next to many new property boundaries) should still exist today. The attempt was made to identify the property corners, and to use those to geo-reference the historical maps. The coordinates of the property corners in Figure 5 are provided in the MGA94 coordinate system. However, careful investigation has shown that the corner points highlighted in red in Figure 5 are incorrect, and that, therefore, not always did a simple subdivision take place. Therefore, this method has been discharged.

Alternatively, the river and its topography are inspected in up-todate airborne images and compared with the information provided in the historical maps, as well as the redrawn map based on the field sheets. It became clear that there are areas of the river which are unlikely to have changed. Therefore, it was decided to use these common points for coordinate transformations which are reviewed in the next section.

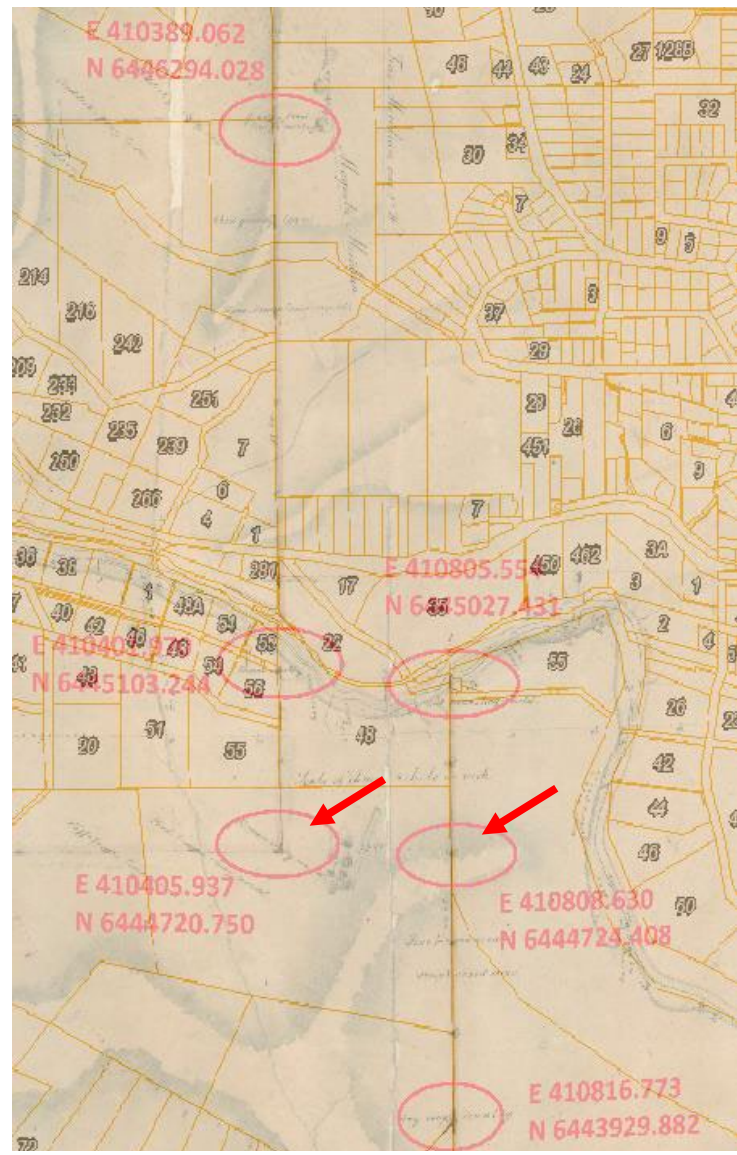

Figure 5: Historical map overlaid with today's cadastral information in the attempted to geolocate the historical map.

\section{METHODOLOGY}

In order to assess the accuracy of the re-drawn map as well and to perform the geo-referencing process, several different transformations have been utilised. Also, an overview of the visualisation methods and the technology used is provided.

\subsection{Similarity and affine transformation}

The similarity and affine transformation equations are given in (1) and (2) respectively.

$\left[\begin{array}{l}X_{T} \\ Y_{T}\end{array}\right]=\left[\begin{array}{ll}\lambda \cos \theta & \lambda \sin \theta \\ -\lambda \sin \theta & \lambda \cos \theta\end{array}\right]\left[\begin{array}{l}X_{I} \\ Y_{I}\end{array}\right]+\left[\begin{array}{l}\Delta x \\ \Delta y\end{array}\right]$

$\left[\begin{array}{l}X_{T} \\ Y_{T}\end{array}\right]=\left[\begin{array}{cc}\cos \theta & \sin \theta \\ -\sin \theta & \cos \theta\end{array}\right]\left[\begin{array}{cc}1 & \sin \delta \\ 0 & \cos \delta\end{array}\right]\left[\begin{array}{cc}S_{x} & 0 \\ 0 & S_{y}\end{array}\right]\left[\begin{array}{c}X_{I} \\ Y_{I}\end{array}\right]+\left[\begin{array}{l}\Delta x \\ \Delta y\end{array}\right]$

In (1) and (2), $X_{T}$ and $Y_{T}$ are the coordinates of the target system (e.g. MGA94 system) while $X_{I}$ and $Y_{I}$ are the coordinates of the input system (e.g. the river local system of the re-drawing map). Both transformations account for translation parameters $(\Delta x$ and $\Delta y)$ as well as for a rotation $(\theta)$. While equation (1) only considers for one scale factor $(\lambda)$, equation (2) considers a scale factor for x and y $\left(S_{x}\right.$ and $\left.S_{y}\right)$. Furthermore, equation (2) also considers a skew angle $(\delta)$. The parameters can be determined using common points in a least square adjustment. Afterwards, the parameters can be applied to all other points in order to perform the transformation of these points from the input system into the target system. For the process of finding the transformation parameters as well as for the transformation of points a self-implemented MATLAB code was utilised.

\subsection{Projective transformation}

Projective transformation is a non-linear transformation method that implicitly estimates the scale, rotation and tilt of coordinate system planes. While the similarity transformation solves for 4 parameters, and the affine transformation solves for 6 parameters, the projective transformation solves for $8\left(a_{1}, a_{2}, a_{3}, b_{1}, b_{2}, b_{3}, c_{1}\right.$, $c_{2}$ ) parameters using the equations (3) and (4).

$x_{T}=\frac{a_{1} x_{I}+a_{2} y_{I}+a_{3}}{c_{1} x_{I}+c_{2} y_{I}+1}$

$y_{T}=\frac{b_{1} x_{I}+b_{2} y_{I}+b_{3}}{c_{1} x_{I}+c_{2} y_{I}+1}$

For the project transformation the implementation in QGIS (version 3.2) (Lennert, 2017) has been utilised.

\subsection{Thin Plate Spline (TPS) transformation}

Referring to a thin sheet of metal, this transformation has its physical analogy involving bending of the metal sheet. Hence, it is not a ridged transformation like the transformation introduced previously. TPS enables local deformations in the data, implying a penalty involving the smoothness of the fitted surface. Therefore, this spline-based technique for data interpolation and smoothing is especially useful when very low-quality originals are being georeferenced. The residuals for all used control points are nearly zero, as the thin plate is made fitting through those points. For further details see Duchon (1976).

For the TPS transformation the implementation in QGIS (version 3.2) (Lennert, 2017) has been utilised.

\subsection{Visualisation technique and technology}

Firstly, the re-drawn map, historical map and aerial photography are combined with a digital elevation model (DEM) of the area derived from LiDAR at a $5 \mathrm{~m}$ resolution from 2015 (GeoScience 
Australia, 2020). These maps are then overlaid within the Unity application (Unity Technologies, 2020) to develop the visualisation. Additional functionality was implemented and included:

- $\quad$ Adding and changing the height scale of the DEM data dynamically with different maps

Changing the different maps' visibility dynamically

Automating a camera viewing path over areas

Water, Texture and Lighting adjustments to enhance realism

Then, a second visualisation was also implemented which took the three maps and DEM data and was used to create a point cloud of each map overlaid on the DEM data. This was achieved utilising Maya 2019 (Autodesk) to create a mesh object textured with each map which was then converted to a point cloud using Present (Euclideon Holographics). In addition, Open Street Map data (also projected using MGA94) has also been used.

The visualisations are presented in the Curtin University HIVE facility with three different display screen technologies.

Firstly, a high-resolution Tiled Display is used to view the visualisation at a screen resolution of $7860 \times 3240$ pixels (24 MPix) with a screen area of $10.028 \mathrm{~m}^{2}$. The Tiled Display allows for the most visually detailed image to allow direct comparison between the different maps to look for variations and some of the historical hand drawn details due to the possibility to present fine detail clarity on the map data.

Secondly, a curved Cylinder Display with stereoscopic projectors with time-sequential shutter glasses was used. This is $5048 \mathrm{x}$ 1200 pixels resolution with a screen area of $37.699 \mathrm{~m}^{2}$. The Cylinder Display provided stereoscopic rendering on a very large surface. This provided the most accessible perception of the terrain surface comparatively to the mapped data. Furthermore, the Cylinder Screen adds stereoscopic with time sequential shutter glasses, which allows three-dimensional perception of the topographical data with the different maps aligned for comparison.

Thirdly, there was the Holographic Table (Euclideon Holographics) with four stereoscopic projectors underneath and IR tracked time-sequential shutter glasses for two users with stereoscopic views of the point cloud data with corrected projection. It allows the two users with separate $3 \mathrm{D}$ glasses to view different views of the terrain which are adjusted to their viewing point to give highly accurate representation of the map data in stereoscopic 3D. The table has a resolution of $1440 \times 1440$ pixels and a screen area of $1.6129 \mathrm{~m}^{2}$.

\section{RESULTS AND DISCUSSION}

In this section, firstly the transformation of the re-drawn map into today's geodetic datum is performed. Then follows discussion of how redrawing maps based on the original field sheets compares with the scanned historical maps. The section closes with the visualisation of the data.

\subsection{Transformation of the re-drawn map based on the original field sheets to today's geodetic datum}

Firstly, the previously mentioned magnetic declination of 5 degrees has been applied to the re-drawn map. Next, common points of suspected unchanged locations are identified by comparison the re-drawn map and the current paths of the river in OSM. Overall, 17 common points have been identified and utilised in a similarity (equation 1) and affine transformation (equation 2) in order to define the transformation parameters.
As the redrawn map is in a local system with a map scale factor of one, and considering the map scale factor of 0.9997156 for the MGA system in which the today's maps are defined, it is assumed that a scale factor of this dimension can be found. Furthermore, it is assumed that there is no large rotation as the magnetic declination has been applied already. There is no meaning of the magnitude to the translation vector as the first point of the redrawn map was given arbitrary coordinates which, by purpose, have been selected to be close to the true location.

The results of the affine and similarity transformation are presented in Table 1.

\begin{tabular}{|c|c|c|}
\hline Parameters & Similarity & Affine \\
\hline Rotation (Minutes) & 27 & 22 \\
\hline Scale & 0.999949 & $\sim$ \\
\hline$\Delta x(\mathrm{~m})$ & 27.0611 & 27.0611 \\
\hline$\Delta y(\mathrm{~m})$ & -65.7418 & -65.7418 \\
\hline$S_{x}$ & $\sim$ & 0.9934 \\
\hline$S_{y}$ & $\sim$ & 1.0018 \\
\hline Skew (Minutes) & $\sim$ & -16 \\
\hline
\end{tabular}

Table 1: The similarity and affine transformation parameters for the geo-location of the re-drawn map based on the historical field sheets.

While the magnitude of the translation is not important, the translation parameters are similar for both translations. The scale parameters of both transformations are also similar and similar to the predicted scale factor of 0.9997156 .

The calculated rotation angle using the affine transformation is slightly lower than the rotation angle calculated using the similarity. The difference is 5 minutes and the magnitude of the rotation is around 25 minutes, and therefore below the tolerance of the used compass for the angle readings. The calculated Root Mean Squared Errors (RMSE) of the 17 common points are $21.7 \mathrm{~m}$ and $21.1 \mathrm{~m}$ for similarity and affine respectively are high.

Next, all other survey points (1376 points) including stations, river boundary and points of interest are transformed from the local system of the re-drawing map to the MGA94 using the calculated similarity and affine transformation parameters. The RMSE of the 1376 points geo-located by similarity and affine parameters are $7.6 \mathrm{~m}$ in $\mathrm{X}$ and $3.5 \mathrm{~m}$ in $\mathrm{Y}$ between them. These differences are insignificant considering the accuracy of the used common points, and the fact that river points may have changed in the last 180 years. Thus, both methods are sufficient to obtain accurate geo-located coordinates. Figure 6 shows a small section of the river points from the re-drawing map (black dots) overlaid on OSM. Some of the differences are very large, and maybe can be explained with an actual change of river points.

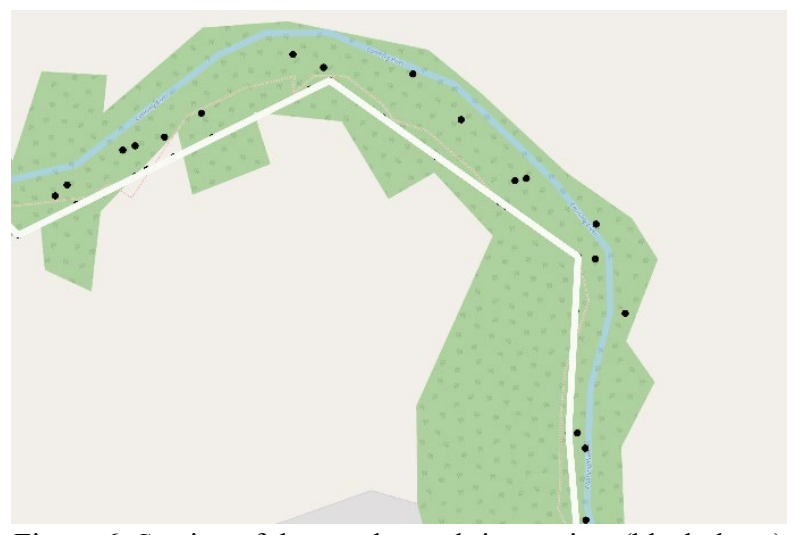

Figure 6: Section of the geo-located river points (black dotes) on OSM. 
As a conclusion, the absolute accuracy (positional accuracy) of the re-drawn map to today's geodetic datum is in the magnitude of less than $10 \mathrm{~m}$.

\subsection{Transformation of historical maps to geo-referenced re- drawn map}

Next, the aim is to compare the relative accuracy of the re-drawn map to the historical maps. Affek, A. (2013) suggest that if the historical maps are based on geodetic measurements (e.g. triangulation networks) and a geocentric geographic coordinate system with graticules or measured grids is used, then usually a similarity of affine transformation is applied. While both are not true for the re-drawn map and the historical map, both should be in the same coordinate system. Therefore, for the transformation of those datasets, the similarity transformation is applied. However, instead of applying the affine transformation the projective transformation is used to account for any errors which maybe have been introduce during the scanning process.

Point-labels of the re-drawn map and the historical maps are identical, which made it easy to define common points. Overall, for each historical map 10 common points have been identified and utilised a similarity transformation (equation 1) and a projective transformation (equation 2).

Due to problems with the scanning process the maps are not to scale. Furthermore, the scanned images have a pixel coordinate system and the re-drawn geo-located maps is in the metric system. Hence, large scaling numbers are suspected. Furthermore, the historical maps have not been scanned with north to the top of the page; so the rotation angles also do not provide with any further information. As none of the transformation parameters provide any valuable information, the focus next is only on the residual plots.

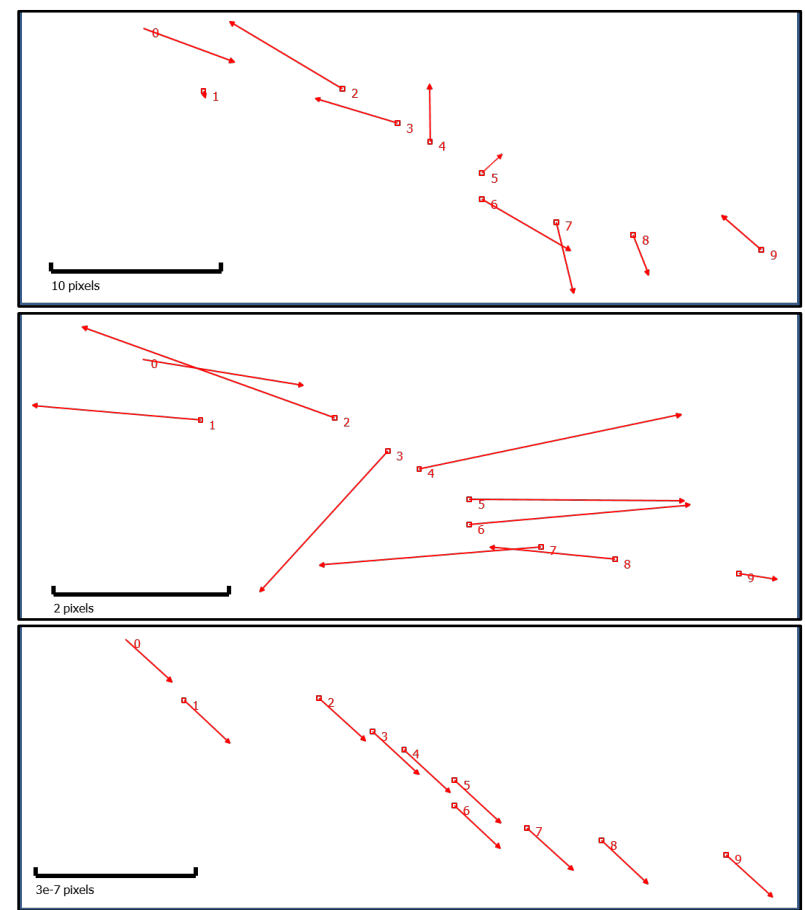

Figure 7: Residual plot for the transformation of the historical map using a similarity transformation (top), projective transformation (middle) and Thin Plate Spline (TPS) transformation (bottom). 1 pixel is approximately $1 \mathrm{~m}$.

The residual plots for the similarity transformation is provided in Figure 7 (top), and for the projective transformation in Figure 7 (middle). Please note that the provided scales for the plot are different. Overall, the magnitude of the residuals of the projective transformation is much smaller. An important aspect is that the residuals are all small, similar and random. Therefore, no systematic trend has been detected.

The magnitudes of the residuals are also clearly visible in the calculated RMSE values in Table 2. The RMSE of the projective transformation is half of the RMSE of the similarity transformation.

\begin{tabular}{|c|c|c|c|}
\hline & $\begin{array}{c}\text { RMSE in } \\
\mathrm{X}(\mathrm{m})\end{array}$ & $\begin{array}{c}\text { RMSE in } \\
\mathrm{Y}(\mathrm{m})\end{array}$ & $\begin{array}{c}\text { RMSE in } \\
\mathrm{XY}(\mathrm{m})\end{array}$ \\
\hline Similarity & 3.467 & 2.529 & 4.292 \\
\hline Projective & 2.174 & 0.649 & 2.268 \\
\hline
\end{tabular}

Table 2: RMSE for the similarity the projective transformation.

As an additional transformation, the Thin Plate Spline (TPS) transformation is applied. As this transformation creates zero residuals the residual plot in Figure 7 (bottom) is as expected. Please note that the sale bar shows a very small-scale number in this figure.

Overall, it can be concluded that the field notes (re-drawn map) and the historical map fit well which is what has been expected. The relative accuracy is within $5 \mathrm{~m}$.

\subsection{Assessment of the positional accuracy}

The final evaluation step is to evaluate the positional accuracy of the transformed historical maps. For this assessment, an object in the historical maps is located which still (or at least its ruins) exist today. This object is the Nairn House. The house burnt down in the 1930s and therefore only the skeleton of the buildings can be seen today. No other objects shown in the historical maps and field sheets could be identified as still existing today.

Nevertheless, the distances from right corner of Nairn House to its corresponding location in the geo-referenced historical maps created using the similarity, projective and TPS transformation are shown in Figure 8. Today's location of the Nairn corner has been determined using high resolution airborne images provided by EagleView and is also shown in Figure 8.

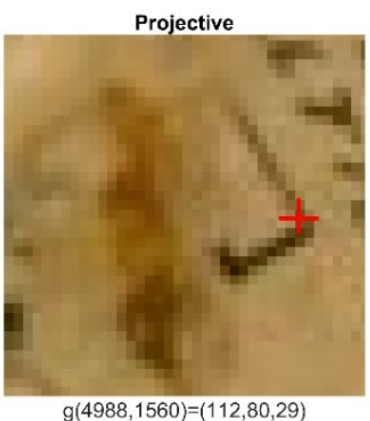

TSP

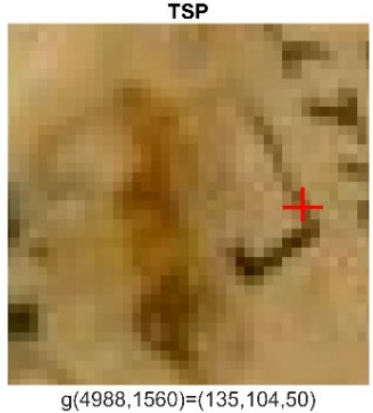

Figure 8: Positional accuracy assessment of the historical maps

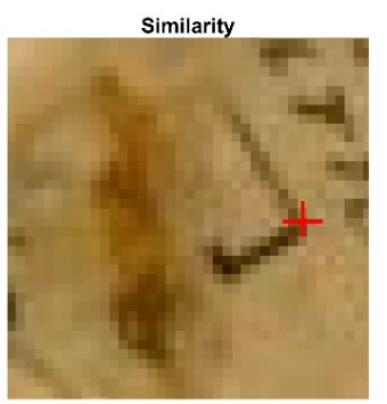

$g(4988,1560)=(114,82,33)$

EagleView image

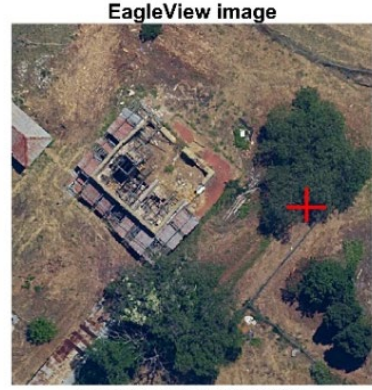

$g(4988,1560)=(9,25,58)$ using Nairn House. 
The distance of the corner of the similarity, projective and TPS transformation map to today are $11.8 \mathrm{~m}, 13 \mathrm{~m}$ and $14 \mathrm{~m}$ respectively. These are very good results and expected based on previous conclusions about the absolute and relative accuracy assessments of approximately $10 \mathrm{~m}$ and $5 \mathrm{~m}$ respectively. It is interesting to note that the similarity transformation produced the best results for this test. This transformation has created the largest residuals in the previous relative and absolute accuracy assessment. Possible explanations are the overfitting of the projective and TSP transformation. However, it must be also pointed out that the historical map was slightly damaged close to the Nairn House which is also visible in Figure 8. This can be another contributing factor for the similarity transformation producing the best result.

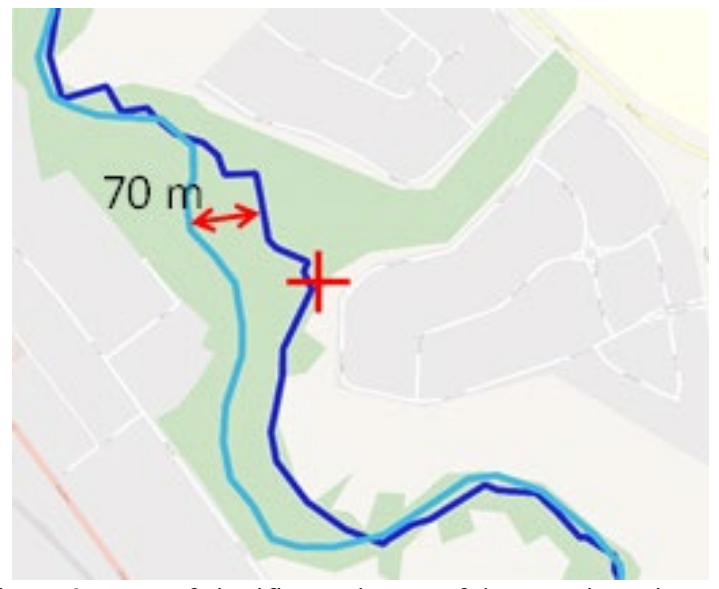

Figure 9: Area of significant change of the Canning River. In dark blue the location of the river in 1841; in light blue the location of the river today (OSM).

DEM

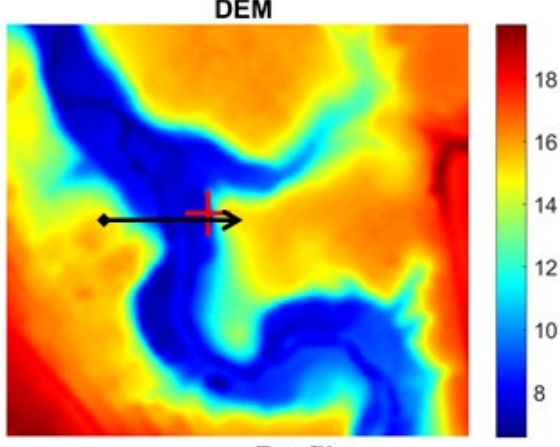

Profile

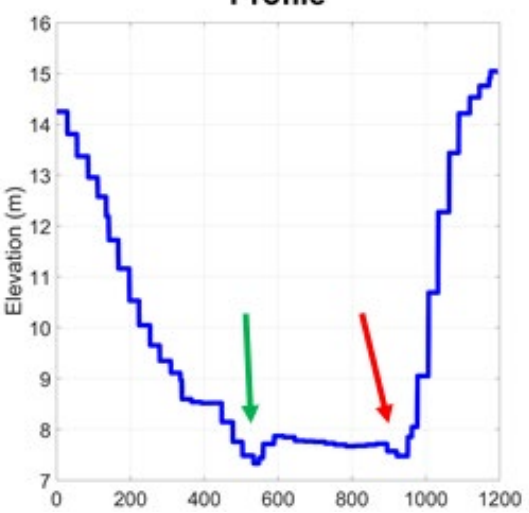

Figure 10: A profile through the DEM for the section indicated by the black arrow is presented in the top figure. In the profile on the bottom: the red and green arrows indicate the old and the current locations respectively of the Canning river.
From the data it is further concluded that there are some significant changes in the watercourse of the Canning River between 1841 and today. Figure 9 shows that the river moved approximately $70 \mathrm{~m}$ as indicated by the red double headed arrow. However, the top and bottom parts in this figure show a strong correspondence with no significant changes.

The Digital elevation model (DEM) of the area shown in Figure 9 is presented Figure 10. A profile for the section indicated by the black arrow in the top figure is presented in the bottom figure. In the bottom profile, the red and green arrows indicate the old and the current locations of the Canning river. This is clearly indicating the movement of the river to left with nearly $70 \mathrm{~m}$. The direction of the watercourse is from the bottom to the top.

Finally, Figure 11 shows the location of the historical maps with EagleView's high resolution images shown in the background, and the location of river taken out of the re-drawn map. Overall, the alignment and accuracy which was calculated for the Nairn House can be confirmed using the overlay.

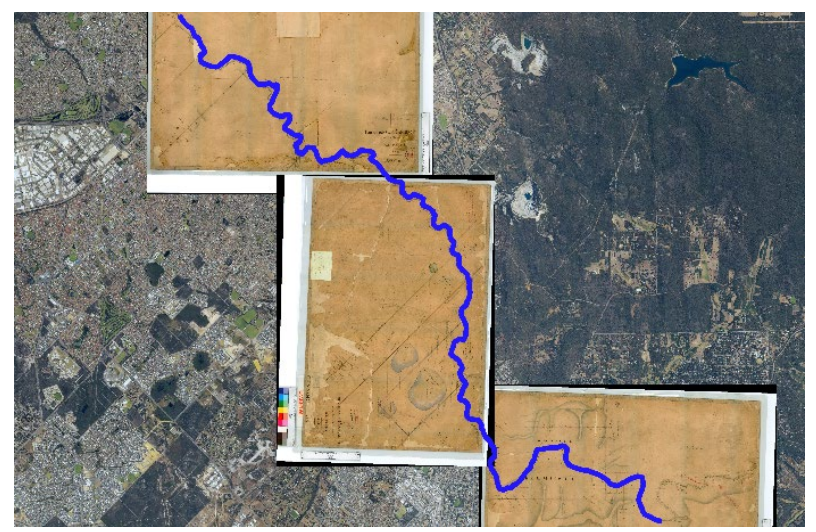

Figure 11: High resolution airborne image with geo-located historical maps.

\subsection{Visualisation}

Visualising the project data has produced several benefits. Firstly, an accessible real time visualisation that is most suitable for public display of the generated and historic maps. Secondly, overlaying the historic content with modern airborne images (MGA94) (provided by EagleView) allows the results to be viewed and interrogated in real time alternating between the modern historical images of the same point. Thirdly, adding the Digital Elevation Data (provided by GeoScience Australia) allows the ability to visualise the historic imagery on top of the historic data and reveal the river path topographically as shown in Figure 2.6 (top figure).

This visualisation shown in Figure 12 (bottom figure) provided a visual and dynamic method to interrogate the data and visualise the conclusions outlined within this paper.

Other benefits:

- $\quad$ The correlation of the historical map with the re-drawn map

- $\quad$ The correlation of the historical map and the re-drawn map to the modern Aerial photography

- The correlation of the historical map to the topology of the terrain for the path of the river

- The correlation of buildings within the modern Aerial photography with historical referenced buildings and the discovery of the Nairn House association. 


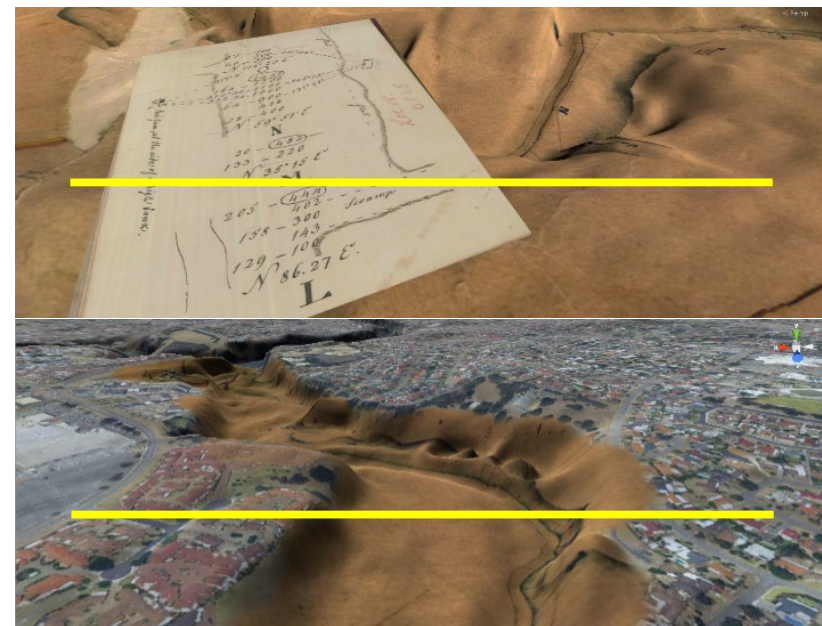

Figure 12: Top: Historical Map visualised with DEM in Unity with associated field sheet. Bottom: Modern aerial photography and historical map combined with DEM in Unity visualisation. The yellow bar indicates the same profile.

The visualisation is built as a custom application which allows for the ease of demonstration and access for the public, collaborators, and other researchers.

\section{CONCLUSION}

In this work, a framework for transforming old survey measurements (bearing and distances) as well as historical maps into today's geodetic referencing system (here: MGA 94) has been introduced. The paper investigated the absolute (app. 12m) and relative accuracy (app. 5m) utilising a number of different transformations. The predicted accuracies could be confirmed utilising the last shown in the 1841 maps and field sheets which still exist today. Furthermore, a pathway for further processing has been outlined in this paper that was implemented to allow non-experts to view the produced results in an accessible visual format.

The results presented here can now be used to reference the field sheets where Preiss drew and named botanical information about the river and to begin mapping the plant biodiversity he recorded. Much more can be done using geo-spatial visualisation to engage publics in new conversations about the river environments. The Preiss field books and maps have a unique role to play. As well as being 180-year-old documents of early land grants to colonists along the Canning River they are also visually compelling images of the millennia-old riverscape and of the abundance of freshwater lakes and fertile wetlands and dense stands of trees and plants. Preiss documented this moment before the staggering environmental transformations of the river began. With several river projects starting in Perth and a new state museum this is an opportune time for the project to show the way using geo-spatial mapping and visualisation.

\section{ACKNOWLEDGEMENTS}

We would like to acknowledge Anna Haebich for introducing Preiss and his maps and field books to the project. Furthermore, we would like to thank EagleView for making the high-resolution airborne images available for this project; the State Record office for their support of tracing historical images and information; and the Curtin CIC and HIVE for supporting the project by providing a scholarship.

\section{REFERENCES}

Affek, A., 2013. Georeferencing of historical maps using GIS, as exemplified by the Austrian Military Surveys of Galicia. Geographia Polonica. Volume 86, Issue 4, pp. 375-390. http://dx.doi.org/10.7163/GPol.2013.30

Balletti, C., 2006. Georeference in the analysis of the geometric content of early maps. e-Perimetron, Vol.1, No. 1, Winter 2006, $32-42$.

Cléry, I., Pierrot-Deseilligny, M., Vallet, B, 2014. Automatic georeferencing of a heritage of old analog aerial photographs. Int. Annals. Photogramm. Remote Sens. Spatial Inf. Sci., II-3, 33- 40. doi:10.5194/isprsannals-II-3-33-2014

GRASS Development Team, 2017. Geographic Resources Analysis Support System (GRASS) Software. Open Source Geospatial Foundation. grass.osgeo.org (20 September 2017).

GeoScience Australia, 2020. Digital Elevation Model (DEM) of Australia derived from LiDAR 5 Metre Grid. https://ecat.ga.gov.au/geonetwork/srv/eng/catalog.search\#/meta data/89644. Last accessed 29/01/2020.

Giordano, S., Le Bris, A., Mallet, C., 2018. Toward automatic georeferencing of archival aerial photogrammetric surveys. Int. Annals. Photogramm. Remote Sens. Spatial Inf. Sci., IV-2, $105-$ 112. https://doi.org/10.5194/isprs-annals-IV-2-105-2018

Herold, H., Roehm, P., Hecht, R., Meinel, G., 2011. Automatically georeferenced maps as a source for high resolution urban growth analyses. Proceedings of the 25th ICA International Cartographic Conference, 5 pages.

Király, G., Walz, U., Podobnikar,T., Czimber, K., Neubert, M., Kokalj, Ž, 2008. Georeferencing of historical maps - methods and experiences. Spatial Information Systems for Transnational Environmental Management of Protected Areas and Regions in the Central European Space, Publisher: Rhombos-Verlag, Editors: Csaplovics, Elmar and Wagenknecht, Stefan and Seiler, Ulrike, 53-63.

Lennert, M., GRASS Development Team, 2017. Addon i.segment.stats. Geographic Resources Analysis Support System (GRASS) Software, Version 7.2, Open Source Geospatial Foundation. grass.osgeo.org/grass $7 /$ manuals/addons/i.segm ent.stats (1 June 2017).

Previtali, M., 2017: GEOPAN AT@S: A brokering based gateway to georeferenced historical maps for risk analysis. Int. Arch. Photogramm. Remote Sens. Spatial Inf. Sci., XLII-2/W5, 583-589. doi:10.5194/isprs-archives-XLII-2-W5-583-2017.

Topcon, 2020. MAGNET Software Suite - Positioning software that puts you in control. https://www.topconpositioning.com/gb/magnet-software-suite Last accessed 28/01/2020).

Unity Technologies, 2020. https://unity.com/ Last accessed $29 / 01 / 2020$

Zhu, L., Erving, A., Koistinen, K., Nuikka, M., Junnilainen, H., Heiska, N., Haggrén, H., 2008. Georeferencing multi-temporal and multi-scale imagery in photogrammetry. Int. Arch. Photogramm. Remote Sens. Spatial Inf. Sci., XXXVII. Part B5, $225-230$. 